\title{
Higher order sequential effects in psychophysical judgments
}

\author{
PETER PETZOLD and GERT HAUBENSAK \\ Justus-Liebig-University of Giessen, Giessen, Germany
}

\begin{abstract}
We found that the depth of sequential effects depends on the judgment task. An experiment with squares indicated that stimulus-response pairs up to two trials back were included in the judgment process when subjects were required to make category judgments of size, whereas only the immediately preceding event was incorporated when subjects were making magnitude estimations. In the case of category judgment, interactions between the current stimulus and prior stimuli as well as configural effects indicated that events one and two trials back meet an equivalent function in the judgment process and that these events may jointly operate in one trial. These findings can be explained by a class of models that assume that the position of preceding stimuli relative to the current stimulus is decisive in the judgment process. The multiple-standards model is a representative of this class according to which there are two types of standards: (1) the endpoints of the range as long-term standards and (2) traces of preceding stimuli as short-term standards.
\end{abstract}

When a series of stimuli is presented and a particular attribute is to be judged, responses depend not only on the current stimulus but also on which stimulus-response pairs occurred on earlier trials. In general, the current response is negatively correlated with the immediately preceding stimuli and positively correlated with the immediately preceding responses (e.g., Jesteadt, Luce, \& Green, 1977; Petzold, 1981; Schifferstein \& Frijters, 1992). Some earlier findings suggest that sequential effects extend over more than one trial. For instance, the analysis of response errors in absolute judgments by Ward and Lockhead (1971) suggests that the depth of sequential effects extends to at least five trials back. However, the effect of remote events could be due to response propagation from trial to trial. For instance, the response on trial $t$ affects the response on trial $t+1$, which, in turn, affects the response on trial $t+2$, and so on. Jesteadt et al. (1977) attempted to explore the depth of sequential effects by using a linear regression model. Analyzing multiple correlations, they found that adding the immediately preceding stimulusresponse pair to the regression equation produced an increment in the multiple correlation, but adding events more than one trial back did not significantly increase the correlation coefficient. From this finding they concluded that sequential effects are limited to the stimulus-response event of the immediately preceding trial. Staddon, King, and Lockhead (1980), however, argued that at least the preceding two trials back are involved. Their arguments are based on the finding that the response error in absolute judgments is positively correlated with the stimuli of lag 1 ,

This study was supported by the Deutsche Forschungsgemeinschaft (Ha 936/6). Correspondence should be addressed to P. Petzold, Fachbereich Psychologie, Universität Giessen, Otto-Behaghel-Straße 10, D-35394 Giessen, Germany (e-mail: petzold-jena@t-online.de). but negatively correlated with the stimuli of lag 2 . Such a crossover cannot be accounted for by a model that incorporates only events one trial back. Similarly, in mixedmodality psychophysicalscaling, Ward $(1985,1986)$ found sequential effects for lags up to four trials. Possibly, the depth of sequential effects depends on the nature of the judgment task. Staddon et al. referred to studies on absolute judgments, whereas Jesteadt et al. focused on three experiments on magnitude estimation.

If events more than one trial back affect the judgment of the current stimulus, what is the nature of such higher order sequential effects? More specifically, are effects of different order mediated by different processes? Ward and Lockhead (1970) argued that the immediately preceding event serves as a comparative standard, whereas the effect of events more than one trial back is mediated by other processes. Alternatively, one could argue that events further back can also function as standards in the same way as the immediately preceding event (Haubensak, 1992a).

The present study was designed to investigate higher order sequential effects, especially concerning two questions. First, is the depth of sequential effects dependent on the task? Judgment processes in magnitude estimation and in category judgment might be different and cause different depths. Second, if events further back are incorporated into the judgment process, do they meet the same function as the immediately preceding event?

The article begins with a discussion of models that allow one to derive more specific hypotheses concerning higher order sequential effects, especially interactions of prior events.

\section{Models of Sequential Effects \\ Previous events as a frame of reference. A number of} models share the assumption that a relation between the 
internal representation $X(t)$ of the stimulus to be judged and the trace $X^{*}(t-1)$ of the preceding stimulus determines a relation between the current response $r(t)$ and the preceding one $r(t-1)$. This idea is specified in the model of category judgments proposed by Holland and Lockhead (1968). In this model, the distance $X(t)-$ $X^{*}(t-1)$ is taken as a basis for determining to what extent $r(t)$ differs from $r(t-1)$. Another specification of this idea is the response ratio rule by Luce and Green (1974), which postulates that in magnitude estimation, the ratio of responses preserves the ratio of internal representations. This approach can be written as

$$
\frac{r(t)}{r(t-1)}=\frac{X(t)}{X^{*}(t-1)} .
$$

This relation is also an essential constituent of Laming's (1997) theory.

On the other hand, a variety of studies have shown that a stimulus is judged in relation to the series of stimuli as a whole. In particular, the effect of the stimulus range on category ratings suggests that stimuli are judged in relation to the subjective range that is defined by a lower extreme and an upper extreme (Braida \& Durlach, 1988; Braida et al., 1984; Parducci, 1965; Petzold, 1982; Witte, 1975). The essential operation, then, is to compare the representation of the stimulus with one or both of these extremes.

\section{Long-Term and Short-Term Frames of Reference}

To bring together the effect of former events with the influence of the whole set of stimuli, most models share the assumption that two types of contextual referents operate on the judgment process: a long-term frame determined by the set of all stimuli and a short-term frame determined by preceding stimulus-response associations. Whereas the short-term frame is changing from trial to trial, the long-term frame remains relatively constant over time. According to the model by Durlach and Braida (1969), for example, two modes may be applied in the judgment process. In the context-coding mode, judgments refer to the entire stimulus set, whereas in the trace mode the current stimulus is compared with the trace of the previous stimulus. Another specification of the interplay of the two frames was proposed by DeCarlo and Cross (1990), who generalized the response ratio rule for magnitude estimation. It is assumed that the short-term frame, $X(t-1) / r(t-1)$, and the long-term-frame, $X_{o} / r_{o}$, are weighted in judgment as

$$
r(t)=X(t)\left(\frac{r_{o}}{X_{o}}\right)^{1-\lambda}\left(\frac{r(t-1)}{X(t-1)}\right)^{\lambda} v(t) .
$$

The weight $\lambda$ indicates the relative influence of the shortterm frame, with $v(t)$ being an error term. For the special weight $\lambda=1$, all judgments are made in relation to the previous event and Equation 6 reduces to the response ratio rule.
An important idea of the DeCarlo-Cross model is that the relative influence of the two frames can be controlled by the participant. This assumption is supported by the finding that instructions affect the magnitude of sequential effects (DeCarlo, 1994; DeCarlo \& Cross, 1990). The assimilation toward the preceding response is stronger when subjects are instructed to use the preceding event as a referent than when they are oriented to use a fixed reference point.

The relative judgment model by Baird, Berglund, Berglund, and Lindberg (1991) also assumes two types of referents. The extremes of the range are assumed to serve as long-term standards and the trace of the preceding stimulus is assumed to act as a short-term standard. The model specifies which referents are used on a particular trial. The referents closest the current stimulus are applied. When, for example, the current stimulus falls between the preceding stimulus and the maximum stimulus, these two referents are employed. In this way, the model conveys the important idea that the usefulness of referents depends on their distance from the current stimulus.

\section{The Influence of Events Further Back}

Most models of sequential effects take into account only the immediately preceding event. In the models that explicitly include events further back, the following approaches have been suggested. We shall see that the models make different predictions about interactions between events of several lags. These predictions can be tested to determine the viability of the models.

Linear combination of prior events. Some models lead to a linear combination of the effects brought about by prior events. Lockhead and King (1983) specified two types of frames of reference in the following way: A memory for the preceding several stimuli serves as a global frame, whereas the memory of the immediately preceding stimulus acts as a local frame. According to this model, the current stimulus is assimilated to the preceding stimulus and compared with a memory pool of earlier stimuli. This memory pool is considered the mean of the memories of the stimuli that occurred on lags 3-6. What is important is that events further back are incorporated into one standard by an averaging process. Consequently, no interaction between the influence of these events is to be expected.

A model that also leads to an additive influence of prior events is the criterion-setting model (Treisman, 1984; Treisman \& Williams, 1984). This model is formulated in the language of Thurstonian and signal detection theories and specifies mechanisms responsible for setting response criteria. Two mechanisms, stabilization and tracking, shift the criteria in a trial-by-trial fashion. Stabilization shifts all criteria toward the trace of the previous stimulus, producing contrast. Tracking means a shift away from the previous response, which leads to assimilation. The criteria effective on a trial are obtained by a linear combination of the amounts indicated by the two mechanisms and by the events several trials back. Con- 
sequently, the criteria-setting model implies no interaction between stimuli of several lags.

Incorporation of only one prior event into the judgment process: The judgment option model. Another model that also accounts for the influence of events further back is Baird's (1997) judgment option model. According to this model, the response on one trial $t, r(t)$, is a weighted average of the response $k$ trials back, $r(t-k)$, and the judgment $r^{*}$ that would be made if the prior event had no influence. Thus,

$$
r(t)=\omega r(t-k)+(1-\omega) r^{*} .
$$

The weight $\omega$ is given by

$$
\omega=\operatorname{sim}[s(t), s(t-k)]-\operatorname{sim}\left[s_{\max }, s_{\min }\right],
$$

where $\operatorname{sim}[s(t), s(t-k)]$ denotes the similarity between the current and the preceding stimulus and $\operatorname{sim}\left[s_{\max }, s_{\min }\right]$ denotes the similarity between the two extreme stimuli. It is assumed that the largest of the similarity score determines which of the previous responses will be integrated into the judgment. If , for instance, the weight $\omega$ in Equation $3 \mathrm{a}$ is greater for $s(t-2)$ than for $s(t-1)$, then $r(t-2)$ will be incorporated into the judgment and $r(t-1)$ is disregarded. This means that only one past event is involved in the judgment.

From this model it follows that interactions between the effects of events $k_{1}$ and $k_{2}$ trials earlier in the sequence should appear. Consider, for instance, the events one and two trials back. If the distance between $s(t-2)$ and $s(t)$ decreases, then the probability that $r(t-2)$ is substituted into Equation $3 \mathrm{a}$ increases. Consequently, the probability that $r(t-1)$ is included in the averaging process decreases. This means that with a decrease of the distance between $s(t-2)$ and $s(t)$, the correlation between $r(t)$ and $r(t-2)$ increases and the correlation between $r(t)$ and $r(t-1)$ decreases. An analogous result is obtained when the distance between $s(t)$ and $s(t-1)$ is varied. Thus, the model predicts an interaction of the distance between $s(t)$ and $s(t-1)$ and the distance between $s(t)$ and $s(t-2)$ from both the correlations between $r(t)$ and $r(t-1)$ and the correlations between $r(t)$ and $r(t-2)$.

Incorporation of two prior events into the judgment process: The multiple-standards model. The multiple-standards model (Petzold \& Haubensak, in press) combines and specifies ideas partly included in former models, especially the notion of long- and shortterm frames (Baird et al., 1991; DeCarlo \& Cross, 1990; Durlach \& Braida, 1969; Petzold, 1981) and the assumption that two prior stimuli may serve as standards in one trial (Haubensak, 1992a). The multiple-standards model is a generalization of range models according to which the judgment of a stimulus is determined by the proportion of the contextual range lying below it (Durlach \& Braida, 1969; Parducci, 1965; Volkmann, 1951; Witte, 1975). More specifically, range models assume that judgments are based on a decision continuum, $Z$, given by

$$
Z=\frac{X-X_{\min }}{X_{\max }-X_{\min }}
$$

where $X$ is the subjective value of the stimulus and $X_{\min }$ and $X_{\max }$ are minimum and maximum subjective values of the range defined by the extremes. Put another way, judgments refer to two internal standard-response pairs: $X_{\min }$ associated with the lowest response $r_{\min }$ and $X_{\max }$ associated with the highest response $r_{\max }$. The relative deviation of the current response from the lowest response,

$$
\frac{r-r_{\min }}{r_{\max }-r_{\min }},
$$

is then determined by the relative deviation of $X$ from $X_{\text {min }}$ given by the value of $Z$.

The range model can be generalized by assuming that not only the extremes of the range but also other standards may contribute to the formation of the subjective range. In particular, previous stimulus-response associations may serve as referents for the judgment. Then we have two types of standards: the extremes of the range as longterm standards and the traces of previous stimuli as shortterm standards. Whereas the comparatively stable longterm standards are assumed to be permanently available in memory during an experiment, short-term standards will be forgotten and substituted by later ones after some trials. The standards used on a particular trial depend on their position relative to the representation $X$ of the current stimulus. The next lower standard is assumed to serve as a lower referent $X_{1}$ and the next higher standard serves as an upper referent $X_{u}$. The process described by Equations $4 \mathrm{a}$ and $4 \mathrm{~b}$ is generalized by a process in which the value of

$$
\frac{r-r_{l}}{r_{u}-r_{l}}
$$

is determined by the value of

$$
Z=\frac{X-X_{l}}{X_{u}-X_{l}}
$$

where $r_{1}$ and $r_{u}$ are the responses associated with the standards $X_{1}$ and $X_{u}$. The multiple-standards model differs from other models (Baird et al., 1991; DeCarlo \& Cross, 1990; Durlach \& Braida, 1969) that also assume more than one standard by the assumption that $t w o$ previous stimuli may be included in the judgment process on one trialone as the lower standard and one as the upper standard.

From this assumption it follows that interactions between the influence of prior events should occur that are determined not only by distances between the current stimulus and prior stimuli, but also by their positions relative to each other. To illustrate this configural effect, let us consider the special case that only the events one and two trials back are available in short-term memory. Two configurations are compared in Figure 1 in which the distances between $s(t)$ and $s(t-1)$ as well as between $s(t)$ and $s(t-2)$ are equal, but the position of $s(t-2)$ relative to $s(t)$ and $s(t-1)$ differs.

Whereas $s(t-1)$ and $s(t-2)$ lie on the same side of $s(t)$ along the dimension to be judged in Configuration A, they are located on different sides in Configuration B. According to the principle of closest standards, this varia- 


\section{Configuration A}

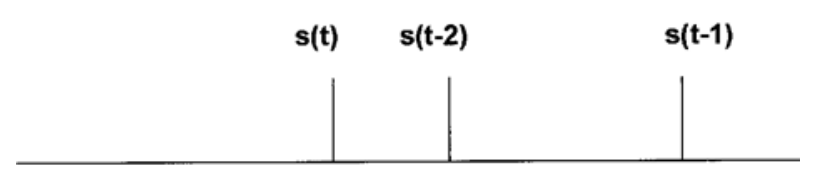

\section{Configuration B}

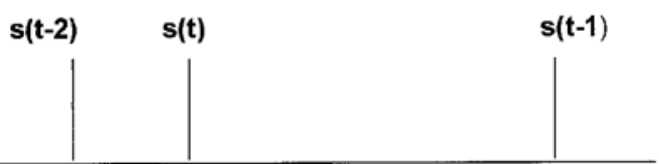

Figure 1. Configurations of $s(t), s(t-1)$, and $s(t-2)$ that differ in the position of $s(t-2)$ relative to $s(t)$ and $s(t-1)$. In Configuration A, $s(t-2)$ lies between $s(t)$ and $s(t-1)$, and in Configuration $B$, it lies outside.

tion of the position leads to different choices of the lower standard $X_{1}$ and the upper standard $X_{u}$.

$$
\begin{aligned}
& \text { Configuration A: } X_{\min }<X(t)<X(t-2)<X(t-1)<X_{\max } \\
& X_{1}=X_{\text {min }} \\
& X_{u}=X(t-2)
\end{aligned}
$$

Configuration B: $X_{\min }<X(t)<X(t-2)<X(t-1)<X_{\max }$ $X_{1}=X(t-2)$

$X_{u}=X(t-1)$.

One can see that the event one trial back is not included in the judgment process in Configuration A. Therefore, the correlation between successive responses should be zero, whereas a correlation greater than zero should exist between $r(t)$ and $r(t-2)$. In Configuration $\mathrm{B}$, however, both $X(t-1)$ and $X(t-2)$ serve as internal standards, and a significant correlation is to be expected between $r(t)$ and $r(t-1)$ as well as between $r(t)$ and $r(t-2)$.

In sum, models of higher order sequential effects differ in predictions concerning the interaction between the effects of events several trials back. We can distinguish three types of models of higher order sequential effects. The first type assumes a linear combination of the effects of several trials and no interaction between the events. The second type (e.g., the judgment option model) predicts an interaction between the events several trials back, where only distances between prior stimuli and the current stimulus are decisive for interactions. The third type of model implies interactions that are determined not only by distances between stimuli but also by the position of prior stimuli and the current stimulus relative to each other. The multiple-standards model is an example of this type of model, which predicts configural effects.

\section{Other Sources of Sequential Effects}

Although there is some evidence that sequential effects are caused by using prior events as referents for judging current stimuli, other components may also con- tribute to the production of sequential dependencies, particularly guessing behavior and pseudoeffects.

Guessing behavior. When the discriminability of stimuli is low, the internal representation of a stimulus may not be sufficient to determine the response completely. After a stimulus presentation, more than one response might be acceptable. The choice from this subset of responses could be influenced by prior responses. Such a guessing strategy has been suggested by Ward and Lockhead (1971) and Petzold (1981) as a source of sequential effects. In particular, the positive correlation of subsequent responses has been attributed to a tendency to minimize the difference between the value of the previous stimulus and the current response.

Pseudosequential effects. Gregson (1976) and Haubensak (1992b) have pointed out that mean judgments show sequential dependencies even if the individual data do not when the data are averaged over individuals who tend to judge the stimuli generally high or low. The authors have shown that such pseudosequential effects may even appear in analyzing individual data averaged over an experiment if systematic response shifts occur in the course of the experiment. Such artifacts may be superimposed on sequential dependencies inherent in the judgment process. Because individual judgment behavior shifts more or less in any experiment, one has to examine which part of sequential effects is due to averaging the data. In analyzing the depth of sequential dependencies, we have to test whether or not the effect of remote events is only an artifact.

The primary purpose of the experiment was to determine the depth of sequential effects in category judgment and magnitude estimation. Furthermore, we examined whether interactions between the current stimulus and prior stimuli occurred, and if so, whether configural effects appeared. The objective was to determine what type of model is appropriate to describe higher order sequential effects.

\section{METHOD}

\section{Stimuli}

The stimuli were squares differing in size. The set of stimuli consisted of 12 squares with sides ranging from 50 to $83 \mathrm{~mm}$ in steps of $3 \mathrm{~mm}$. They were presented on a 17 -in. computer screen.

\section{Participants}

There were 41 paid adult observers, each of whom was naive with respect to the experimental procedure. Participants performed in one of two judgment tasks; 31 participated in category judgment and 10 in magnitude estimation.

\section{Procedure}

After the printed instructions had been read by the individuals (together with the experimenter, who read it aloud), a random sequence of stimuli was presented on a computer screen.

In the category judgment task, the participants were asked to judge the size of the squares on a five-category scale by pressing the respective key on the computer keyboard. In the magnitude estimation task, at the beginning of the series, Square 6 was presented as a standard stimulus, to which the Modulus 10 was assigned. Partic- 
Table 1

Partial Correlations Between the Current Response and Prior Stimuli and Responses for Category Judgment

\begin{tabular}{ccc}
\hline lag $k$ & $\rho[r(t), s(t-k)]$ & $\rho[r(t), r(t-k)]$ \\
\hline 1 & $-.198_{\mathrm{a}}$ & $.272_{\mathrm{a}}$ \\
2 & $-.136_{\mathrm{b}}$ & $.128_{\mathrm{b}}$ \\
3 & $-.097_{\mathrm{c}}$ & $.070_{\mathrm{c}}$ \\
4 & $-.076_{\mathrm{c}}$ & $.061_{\mathrm{c}}$ \\
5 & $-.088_{\mathrm{c}}$ & $.080_{\mathrm{c}}$ \\
6 & $-.057_{\mathrm{c}}$ & $.061_{\mathrm{c}}$ \\
\hline
\end{tabular}

Note-All values are significant at the .01 level. Values in the same column that do not share a common subscript differ at the .01 level. $s(t)$, the current stimulus; $r(t)$, the current response; $s(t-k)$, the stimulus $k$ trials back; $r(t-k)$, the response $k$ trials back.

ipants were requested to judge the subsequent squares relative to this standard. They were told that they should choose the number 20 if they had the impression that the current square was twice as large as the standard, and the number 5 if they felt that the current square was half the size of the standard. Again, participants typed their response on the computer keyboard.

In both judgment tasks, the next square appeared $1 \mathrm{sec}$ after a key had been pressed. There was no feedback. The sessions consisted of three blocks lasting for about $10 \mathrm{~min}$ each. There were 200 trials per block. Between the blocks there was a rest of about $5 \mathrm{~min}$. Observers were instructed to respond as fast as possible and to rely on their first impression.

\section{RESULTS}

\section{Depth of Sequential Effects}

To measure the influence of prior events, individual correlations between $r(t)$ and $s(t-k)$, as well as those between $r(t)$ and $r(t-k), k=1,2, \ldots 6$, were computed. When the correlation between $r(t)$ and one of the variables was calculated, all other variables were partialed out. The results averaged over the individuals are presented in Tables 1 and $2 .{ }^{1}$ As can be seen, the values decrease up to three trials back for category judgment, whereas they decrease up to two trials back for magnitude estimation and remain approximately constant on later trials. We can suppose that the constant level for remote trials is produced by pseudosequential effects. To examine this assumption, we tested whether the magnitude of individual response shifts was sufficient to account for the values of correlations.

We took the following steps. First, individual mean judgments were calculated for each stimulus within each of the three blocks of the experiment separately. Second, to produce stimulus-response series without sequential effects, these mean judgments were used as scale values in a computer program based on a Thurstone-like model. This was done for each individual and for each block of the experiment. Third, the series from the three blocks were combined for each individual to calculate partial correlations between $r(t)$ and $r(t-k)$ that were due to drifts of judgment scale.

The empirical data and pseudosequential effects averaged over the participants are represented in Figure 2. The curves differ for category judgment and magnitude estimation. In the case of category judgment, the empir- ical correlations for lags 1 and 2 have higher values than those produced by pseudosequential effects. For higher lags, the values found in the experiment correspond roughly to the magnitude of the correlations produced by a drift of scale. From these results it can be concluded that sequential effects up to lag 2 are caused by the judgment process and that the correlations for events more than two trials back result from pseudosequential effects. In the case of magnitude estimation, the empirical value is higher than the calculated pseudosequential effect only for lag 1, and the correlations reach the level of pseudoeffects already at lag 2 . These findings suggest that the depth of sequential effects is different for category judgment and magnitude estimation. Whereas events up to lag 2 seem to be included in the judgment process for category judgments, only events of lag 1 appear to serve as referents for magnitude estimation.

This conclusion is further supported by relationships between individual empirical correlations and individual values of the calculated pseudoeffects.

1. The asymptotic level of correlations for events more than two trials back and the level of calculated pseudoeffects was correlated over participants. The correlations were $\rho=.51(p<.01)$ for category judgment and $\rho=$ $.84(p<.001)$ for magnitude estimation. These findings suggest that the correlations between $r(t)$ and $r(t-k)$ for remote events are a consequence of averaging judgments drifting upward or downward during the experiment.

2 . The correlation between successive responses, $r(t)$ and $r(t-1)$, shows no relation to the level of the calculated pseudoeffects. The correlations over participants are $\rho=.23(p=.21)$ for category judgment and $\rho=-.05$ $(p=.79)$ for magnitude estimation. The missing relation indicates that the correlation between successive responses and the calculated pseudoeffects comes from different sources.

3. The correlation between $r(t)$ and $r(t-2)$ and the calculated pseudoeffects shows a different relationship for category judgment and magnitude estimation. Whereas there was no significant correlation over participants for category judgment $(\rho=-.20, p=.59)$, a marginally significant correlation was found for magnitude estimation $(\rho=.62, p=.07)$. This finding supports the conclusion that, in the case of magnitude estimation, correlations between the current response and the response two trials

Table 2

Partial Correlations Between the Current Response and Prior Stimuli and Responses for Magnitude Estimation

\begin{tabular}{ccc}
\hline lag $k$ & $\rho[r(t), s(t-k)]$ & $\rho[r(t), r(t-k)]$ \\
\hline 1 & $-.178_{\mathrm{a}}$ & $.281_{\mathrm{a}}$ \\
2 & $-.060_{\mathrm{b}}$ & $.059_{\mathrm{b}}$ \\
3 & $-.089_{\mathrm{b}}$ & $.082_{\mathrm{b}}$ \\
4 & $-.061_{\mathrm{b}}$ & $.078_{\mathrm{b}}$ \\
5 & $-.080_{\mathrm{b}}$ & $.095_{\mathrm{b}}$ \\
6 & $-.065_{\mathrm{b}}$ & $.068_{\mathrm{b}}$ \\
\hline
\end{tabular}

Note-All values are significant at the .01 level. Values in the same column that do not share a common subscript differ at the .01 level. $s(t)$, the current stimulus; $r(t)$, the current response; $s(t-k)$, the stimulus $k$ trials back; $r(t-k)$, the response $k$ trials back. 

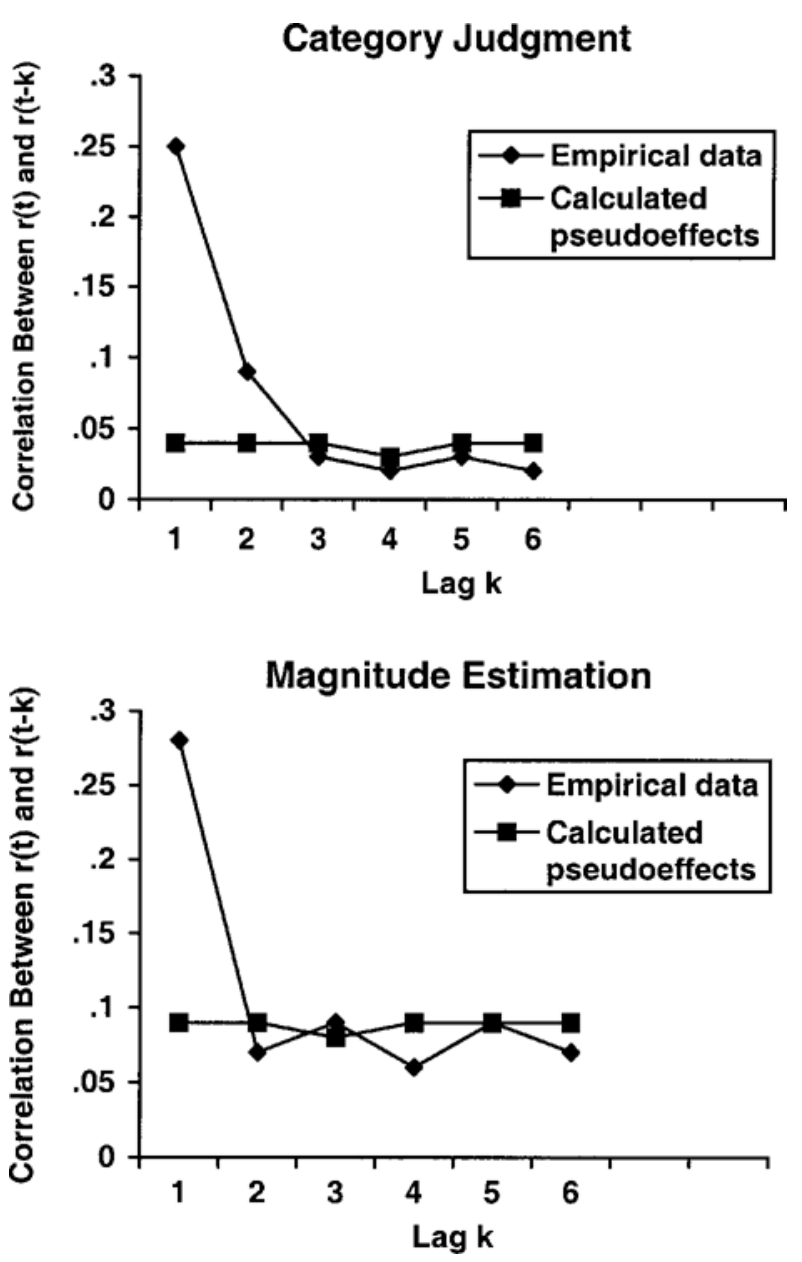

Figure 2. Empirical partial autocorrelations of responses as a function of lag and values for pseudosequential effects. The calculation of pseudosequential effects is described in the text.

back are produced by pseudoeffects. In the case of category judgment, however, they are caused by the judgment process.

\section{Interactions Between the Current Stimulus and Prior Stimuli}

To avoid artifacts produced by averaging the data, Schifferstein and Kuiper (1997) proposed correcting for differences in scale usage by calculating standardized responses, which are brought back to a mean of 0 and a standard deviation of 1 . Following this procedure, we determined arithmetic mean, $m$, and standard deviation $\sigma$ of the responses $r$ and calculated standardized responses $r_{s}=(r-m) / \sigma$ for each participant and each part of a session separately. These standardized responses were used to analyze sequential dependencies.

Stimuli were divided into four groups of three adjacent stimuli in order to have adequate sample sizes for each participant. Then, individual mean judgments were entered into a 4 [groups of $s(t)$ ] $\times 4$ [groups of $s(t-1)$ ] $\times 4$ [groups of $s(t-2)]$ analysis of variance (ANOVA) with $r(t-1)$ and $r(t-2)$ as covariates. This was done for category judgment and magnitude estimation separately. For both judgment tasks, there was a significant interaction between $s(t)$ and $s(t-1)[F(9,11456)=2.30, p<$ .05 , for category judgment, and $F(9,3558)=2.27, p<$ .05 , for magnitude estimation]. This interaction corresponds to the well-known phenomenon that the correlation between successive responses will be larger if the distance between successive stimuli is smaller (e.g., Baird, 1997; DeCarlo \& Cross, 1990; Jesteadt et al., 1977). Even more important, the analyses reveal a significant interaction between $s(t)$ and $s(t-2)[F(9,11456)=2.06, p<.05]$ and a three-way interaction between $s(t), s(t-1)$, and $s(t-2)$ $[F(27,11456)=1.80, p<.05]$ for category judgment.

There were no significant interactions for magnitude estimation, however. This finding supports the conclusion that the effective depth of sequential effects is of Order 2 for categorical judgment, but only of Order 1 for magnitude estimation. Moreover, the significant interactions for the category judgment condition suggest that $s(t-1)$ and $s(t-2)$ are included in the judgment process, not independently of each other. This result is in accordance with both the judgment option model and the multiplestandards model.

\section{Configural Effects for Category Judgments}

To examine whether the interactions found for category judgment were determined not only by distances between $s(t), s(t-1)$, and $s(t-2)$ but also by their position relative to each other, we analyzed the following configurations.

$$
\begin{array}{llrl}
\text { 1a } & s(t)=4 \text { or } 5 & \text { 1b } & s(t)=4 \text { or } 5 \\
& s(t-1)=9,10,11, \text { or } 12 & & s(t-1)=9,10,11, \text { or } 12 \\
& s(t-2)=6,7, \text { or } 8 & & s(t-2)=1,2, \text { or } 3 \\
2 \mathrm{a} & s(t)=8 \text { or } 9 & \text { 2b } & s(t)=8 \text { or } 9 \\
& s(t-1)=1,2,3, \text { or } 4 & s(t-1)=1,2,3, \text { or } 4 \\
& s(t-2)=5,6, \text { or } 7 & s(t-2)=10,11, \text { or } 12
\end{array}
$$

In Configurations $1 \mathrm{a}$ and $2 \mathrm{a}, s(t-2)$ is located between $s(t)$ and $s(t-1)$. In this case, according to the multiplestandards model, $s(t-1)$ is not used as a standard when the trace of $s(t-2)$ is available. Therefore, the correlation between $r(t)$ and $r(t-1)$ should be low. In Configurations $1 \mathrm{~b}$ and $2 \mathrm{~b}$, however, $s(t-2)$ is not located between $s(t)$ and $s(t-1)$ and, consequently, $s(t-2)$ will not displace $s(t-1)$ as a standard. Therefore, a higher correlation between $r(t)$ and $r(t-1)$ is to be expected.

To examine the prediction, partial correlations between standardized successive responses were calculated for Configurations $1 \mathrm{a}, 1 \mathrm{~b}, 2 \mathrm{a}$, and $2 \mathrm{~b}$. The results are

Table 3

Correlations Between $\boldsymbol{r}(\boldsymbol{t})$ and $\boldsymbol{r}(\boldsymbol{t}-\mathbf{1})$ for Different Configurations of $s(t), s(t-1)$, and $s(t-2)$ as Described in the Text

\begin{tabular}{ccc}
\hline & $\mathrm{a}$ & $\mathrm{B}$ \\
\hline 1 & $0.16_{\mathrm{a}}$ & $0.28_{\mathrm{b}}$ \\
2 & $0.10_{\mathrm{a}}$ & $0.19_{\mathrm{b}}$ \\
\hline
\end{tabular}

Note-Values in the same row that do not share a common subscript differ at the .1 level. $s(t)$, the current stimulus; $r(t)$, the current response. 
Table 4

Correlations Between $r(t)$ and $r(t-2)$ for Different Configurations of $s(t), s(t-1)$, and $s(t-2)$ as Described in the Text

\begin{tabular}{ccc}
\hline & $\mathrm{A}$ & $\mathrm{B}$ \\
\hline 3 & $-.03_{\mathrm{a}}$ & $.14_{\mathrm{b}}$ \\
4 & $-.06_{\mathrm{a}}$ & $.10_{\mathrm{b}}$ \\
\hline
\end{tabular}

Note-Values in the same row that do not share a common subscript differ at the .05 level. $s(t)$, the current stimulus; $r(t)$, the current response.

represented in Table 3. In accordance with the predictions, the correlation between $r(t)$ and $r(t-1)$ was lower when $s(t-2)$ was located in between $s(t)$ and $s(t-1)$.

When the positions of $s(t-1)$ and $s(t-2)$ change, we obtain the following configurations:

$$
\begin{array}{llrl}
3 \mathrm{a} & s(t)=4 \text { or } 5 & 3 \mathrm{~b} & s(t)=4 \text { or } 5 \\
& s(t-1)=6,7, \text { or } 8 & & s(t-1)=1,2, \text { or } 3 \\
& s(t-2)=9,10,11, \text { or } 12 & & s(t-2)=9,10,11, \text { or } 12 \\
& & 4 \mathrm{~b} & s(t)=8 \text { or } 9 \\
\text { 4a } & s(t)=8 \text { or } 9 & s(t-1)=10,11, \text { or } 12 \\
& s(t-1)=5,6, \text { or } 7 & s(t-2)=1,2,3, \text { or } 4
\end{array}
$$

The predictions are analogous to Configurations 1 and 2 after changing the places of $s(t-1)$ and $s(t-2)$. In Configurations $3 \mathrm{a}$ and $4 \mathrm{a}, s(t-1)$ lies between $s(t)$ and $s(t-2)$. Consequently, only a small correlation between $r(t)$ and $r(t-2)$ is to be expected. In Configurations $3 \mathrm{~b}$ and $4 \mathrm{~b}$, however, $s(t-1)$ is located on the other side of $s(t)$ and, now, $s(t-2)$ can serve as a standard and a higher correlation between $r(t)$ and $r(t-2)$ should occur.

Accordingly, partial correlations between standardized current responses and standardized responses two trials back were calculated. The results are represented in Table 4. Consistent with the predictions, the correlation between $r(t)$ and $r(t-2)$ was lower when $s(t-1)$ was located between $s(t)$ and $s(t-2)$.

\section{The Effect of Range in Category Judgment}

According to the multiple-standards model, the traces of stimuli one and two trials back may jointly serve as standards and determine the actual range. This range is smaller when the stimuli are closer to each other than when they are more distant. Consequently, if mean judgments are considered as a function of current stimuli, this function should be steeper for prior stimuli lying closer to the current stimulus than for the more distant ones. To test this prediction, we calculated individual mean judgments for each of current Stimuli 4-9 and for the four conditions represented in Table 5.

Mean judgments averaged over participants as a function of stimuli are represented in Figure 3. As expected, the curve is steeper for close prior stimuli than for more distant ones. To test this difference, the slope of individual regression lines for the four conditions in Table 5 were calculated. These values were entered into a 2 (distance of prior stimuli) $\times 2$ [position of $s(t-1)$ relative to $s(t)$ : below or above] ANOVA with repeated measures. There was a significant effect of the distance of prior stimuli $[F(1,23)=13.2, p=.001]$. The mean slope was .26 for nearer prior stimuli and .20 for more distant ones.
Additionally, we calculated $d^{\prime}$ for each of the two ranges. The mean value was $d^{\prime}=1.27$ for the nearer prior stimuli and $d^{\prime}=1.11$ for more distant ones. These findings suggest that traces of stimuli one and two trials back actually contribute to the effective range on a trial.

\section{Reaction Times}

The multiple-standards model assumes that both the extremes of the stimulus and the traces of previous stimuli are involved in the judgment process as standards. In this case, analogous regularities should appear for both types of internal standards. For instance, reaction time has been found to decrease in the vicinity of a standard in category judgment. Consequently, reaction time should be comparatively low for stimuli close to the extremes of the range and close to the preceding stimulus. To examine whether there were such trends, mean response times were calculated for category judgments for each individual, each current stimulus, and each distance between the current and the preceding stimuli. Because of the skewed distribution of reaction times, these values were transformed before a statistical analysis was performed. According to Edwards (1968) and Winer (1962), a reciprocal transformation is appropriate for reaction times to diminish the skewness. For the present data, the skewness was 2.37 for the distribution of reaction times and .32 after the reciprocal transformation. Therefore, reciprocal reaction times were entered into a 12 (stimuli from 1 to 12$) \times 6$ [distance between $s(t)$ and $s(t-1)$ from 0 to 6 ] ANOVA. The analysis revealed an effect of both the position of the stimuli within the range $[F(11,2477)=8.7$, $p<.001]$ and the distance between the current and the preceding stimulus $[F(6,2477)=7.0, p<.001]$. There was no interaction between the position of stimuli and the distance from the preceding stimulus $[F(66,2477)=$ $.07, p=.93]$. As can be seen from Figures 4 and 5, the reaction time is minimal at the extremes of the range and increases with an increase of the distance between the current and preceding stimulus. These findings support the assumption that both the extremes of the range and the preceding stimulus serve as internal standards.

\section{DISCUSSION}

\section{Depth of Sequential Dependencies}

For category judgment as well as for magnitude estimation, significant partial correlations between the current response and prior stimuli and responses were found

Table 5

Conditions for Analyzing the Influence of the Range Formed by Previous Stimuli

\begin{tabular}{ccc}
\hline Range & $s(t-1)$ & $s(t-2)$ \\
\hline Narrow & 3 or 4 & 9 or 10 \\
& 9 or 10 & 3 or 4 \\
Wide & 1 or 2 & 11 or 12 \\
& 11 or 12 & 1 or 2 \\
\hline
\end{tabular}

Note- $s(t)$, the current stimulus. 


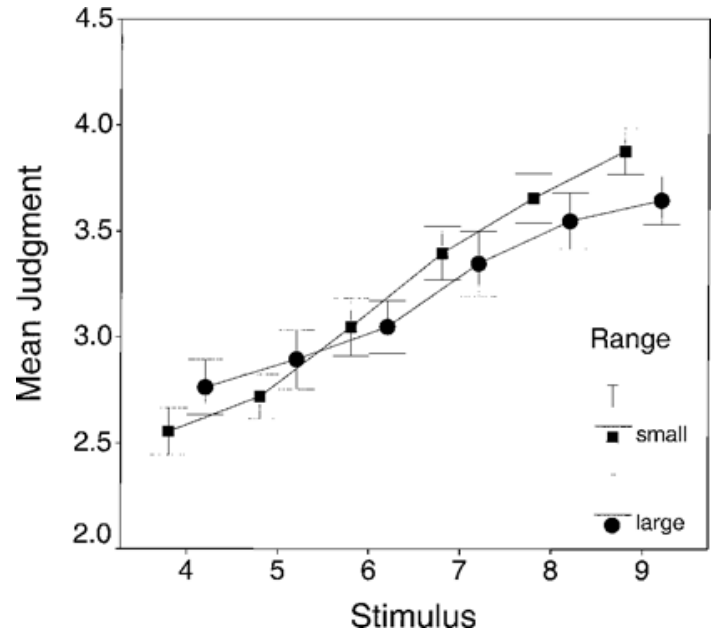

Figure 3. Mean judgments as a function of current stimuli for two ranges constituted by the stimuli one and two trials back.

up to lag 6 . To examine whether these results imply that events more than one trial back are included in the judgment process, we compared empirical correlations with the correlations produced by a drift of the judgment scale across trial blocks. If the actual correlations are greater than the pseudoeffects, one can conclude that sequential dependencies are not just the result of averaging the data. We found that the empirical values were indeed significantly greater up to lag 2 for category judgment, but only up to lag 1 for magnitude estimation.

The conclusion that the depth of sequential dependencies is different for the two judgment tasks is supported by the result that interactions including the event two trials back were significant only for category judgment, and not for magnitude estimation. This finding indicates that the stimulus-response pair two trials back operates in a way equivalent to the immediately preceding one only for category judgment.

Looking for the reasons of the different depth, one might speculate that there are different decision processes in the two judgment tasks. In the case of category judgment, the relative distance of the stimulus from the two endpoints of a subjective range seems to determine the response. Consequently, two prior stimuli can be involved in the formation of the range. For magnitude estimation the relation to one prior event as referent seems to be decisive, as is proposed by the response ratio rule (Luce \& Green, 1974) or the dynamic judgment model (DeCarlo \& Cross, 1990). The exact nature of the difference in the depth of sequential effects needs further clarification. In particular, future research should explore whether the difference found in the present experiment is typical for categorical judgment and magnitude estimation. It is possible that other conditions affect the depth as well.

It is also possible that events more than two trials back are involved in the judgment process with a very low probability. This would lead to very small correlations that cannot be detected in the data because they are washed out by pseudosequential effects. Another point that needs further clarification concerns modality-specific sequential effects. Each modality might have its own depth of sequential effects. Evidence for this speculation comes from studies of Ward $(1982,1985,1986)$ in which stimuli of different modalities were presented in alternating pairs. In these studies, the correlation of $r(t-3)$ and $r(t)$ was dependent on whether or not $s(t-3)$ belonged to the same modality as $s(t)$. A similar result was obtained in an experiment on size estimation of squares where different colors constituted different categories of stimuli (Petzold \& Haubensak, 2000).

\section{Mechanisms Underlying Sequential Effects in Category Judgment}

For category judgment, the event two trials back contributed to the judgment of the current stimulus. Two findings suggest that the influence of the events one and two trials back is mediated by the same mechanism. First, in analyzing mean judgments we found that interactions between $s(t), s(t-1)$, and $s(t-2)$ were symmetric with respect to $s(t-1)$ and $s(t-2)$ (Table 3$)$. Second, the configural effects described in Tables 4 and 5 were equivalent for the stimuli lying one and two trials back. In any case, the partial correlation between the current response and a prior response was higher for those previous stimuli that were closest to the current stimulus along the dimension to be judged.

To account for the equivalent function of events one and two trials back the three types of models described earlier can be considered. The configural effects found in the present experiment favor the third type of model, according to which the relative positions of $s(t), s(t-1)$, and $s(t-2)$ are decisive in the judgment process, and, consequently, interactions between the current stimulus and the preceding stimuli are determined not only by their distances but also by their relative positions. The multiple-

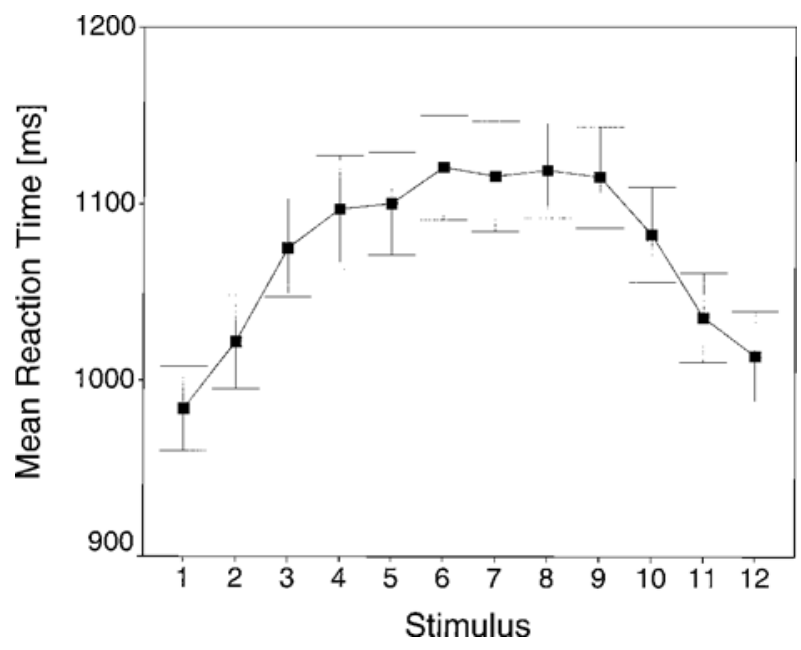

Figure 4. Response time as a function of the current stimulus. 


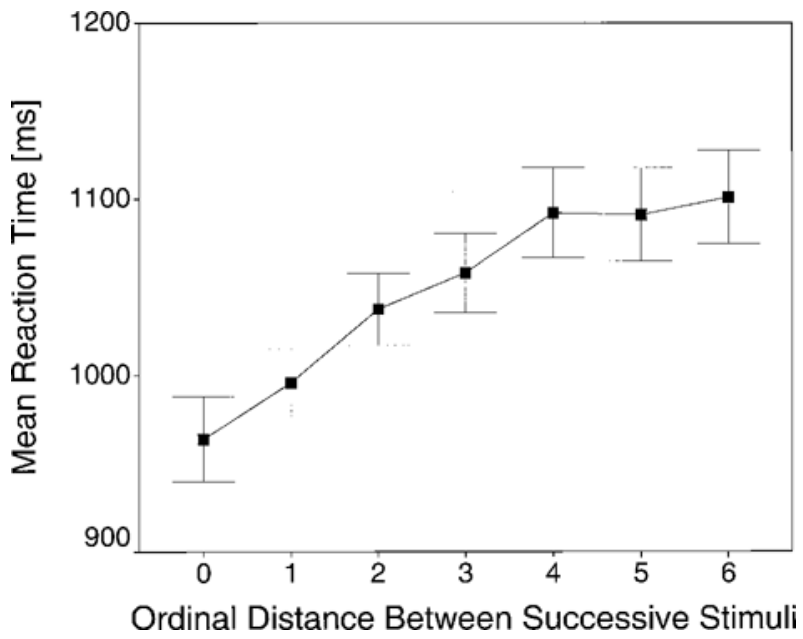

Figure 5. Response time as a function of the distance between the current stimulus and the preceding stimulus.

standards model is a representative of this type of model. The primary assumptions of the multiple-standards model are that (1) the extremes of the range and traces of preceding stimuli may act as standards in an equivalent way, and (2) stimuli one and two trials back may jointly operate as standards. The first assumption is supported by the finding that the response time is comparatively low when the current stimulus comes close to either the endpoints of the range or the preceding stimulus. The second assumption is corroborated by the occurrence of configural effects.

\section{Additional Components}

According to most models, sequential effects are caused by using prior events as referents for judging current stimuli. This does not exclude, however, the possibility that in special cases other components may also contribute to sequential dependencies. For instance, a modality-specific contrast to the preceding stimulus seems to occur in judgments of sweetness intensity (Schifferstein \& Frijters, 1992).

Furthermore, a guessing strategy could also produce sequential dependencies. In case of low stimulus discriminability, more than one response might be acceptable after a stimulus representation. Which of the acceptable responses is finally selected may depend on the preceding response. Ward (1979) suggested in the framework of his fuzzy judgment model that the less informative a stimuli is, the fuzzier is its internal representation. From this it follows that sequential effects should be stronger in case of lower stimulus discriminability. This expectation is consistent with results from earlier studies (e.g., McGill, 1957; Mori \& Ward, 1995; Ward \& Lockhead, 1971).

What is important is that the guessing process is solely response driven and brings about partial correlations only between successive responses but not between the current response and the preceding stimulus. Consequently, the variation of the guessing behavior should af- fect only the correlation between successive responses. This was found in an as yet unpublished supplementary experiment with category judgment of the size of squares. In this experiment, the discriminability of stimuli was deteriorated by a background noise. Whereas the discriminability was $d^{\prime}=0.90$ in the experiment described herein, the values in this other experiment decreased to $d^{\prime}=0.49$ because of the addition of the noise. As a consequence of the lowered discriminability, the partial correlation between successive responses increased to .46, whereas the corresponding value in the condition without noise was .27 (Table 1). There was no significant difference, however, for the partial correlation between the current response and the preceding stimulus ( -.22 and - .18). A similar result has been obtained in a study by Mori (1998). In this study, the stimulus information affected the dependency of the response on $r(t-1)$, but not on $s(t-1)$. Both findings suggest that a response-driven component is affected by the addition of noise. Perhaps this component is a guessing strategy that gains more influence when the stimuli are less discriminable.

An additional response-driven component is also suggested by results found in studies on mixed-modality scaling (Ward, 1982, 1985, 1986). Stimuli of different modalities were presented alternately. The contrast of the current response to the preceding stimulus appeared only within a modality, whereas the assimilation toward the preceding response occurred both within and between modalities. This finding can be interpreted in terms of a guessing mechanism that is independent of the modality. Further research is needed to clarify the joint operation of various components in sequential dependencies.

\section{REFERENCES}

BAIRD, J. C. (1997). Sensation and judgment: Complementarity theory of psychophysics. Mahwah, NJ: Erlbaum.

Baird, J. C., Berglund, B., Berglund, U., \& Lindberg, S. (1991). Stimulus sequence and the exponent of the power function for loudness. Perceptual \& Motor Skills, 73, 3-17.

Braida, L. D., \& Durlach, N. I. (1988). Peripheral and central factors in intensity perception. In G. M. Edelman, W. E. Gall, \& M. Cowan (Eds.), Auditory function (pp. 559-583). New York: Wiley.

Braida, L. D., Durlach, N. I., Lim, J. S., Berliner, J. E., RabinoWITZ, W. M., \& PURKS, S. R. (1984). Intensity perception. XIII. Perceptual anchor model of context coding. Journal of the Acoustical Society of America, 76, 722-731.

DeCARLO, L. T. (1994). A dynamic theory of proportional judgment: Context and judgment of length, heaviness, and roughness. Journal of Experimental Psychology: Human Perception \& Performance, 20, 372-381.

DeCarlo, L. T., \& Cross, D. V. (1990). Sequential effects in magnitude scaling: Models and theory. Journal of Experimental Psychology: General, 119, 375-396.

Durlach, N. I., \& Braida, L. D. (1969). Intensity perception I. Preliminary theory of intensity resolution. Journal of the Acoustical Society of America, 46, 372-383.

EDWARDS, A. L. (1968). Experimental design in psychological research. London: Holt, Rinehart \& Winston.

Gregson, R. A. M. (1976). Psychophysical discontinuity and pseudosequence effects. Acta Psychologica, 40, 431-451.

HAUBENSAK, G. (1992a). The consistency model: A process model for absolute judgments. Journal of Experimental Psychology: Human Perception \& Performance, 18, 303-309. 
Haubensak, G. (1992b). Sequenzeffekte in absoluten Urteilen? Kritisches zur Methode [Sequential effects in absolute judgment: Critical remarks concerning methods]. Zeitschrift für Experimentelle und Angewandte Psychologie, 39,101-113.

Holland, H. K., \& LockHEAd, G. R. (1968). Sequential effects in absolute judgments of loudness. Perception \& Psychophysics, 3, 409-418.

Jesteadt, W., Luce, R. D., \& Green, D. M. (1977). Sequential effects in judgments of loudness. Journal of Experimental Psychology, $\mathrm{Hu}$ man Perception, \& Performance, 3, 92-104.

LAMING, D. (1997). The measurement of sensation. Oxford: Oxford University Press.

Lockhead, G. R., \& King, M. C. (1983). A memory model of sequential effects in scaling tasks. Journal of Experimental Psychology: Human Perception \& Performance, 9, 461-473.

Luce, R. D., \& GReEn, D. M. (1974). The response ratio hypothesis for magnitude estimation. Journal of Mathematical Psychology, 11, 1-14.

McGILL, W. J. (1957). Serial effects in auditory threshold judgments. Journal of Experimental Psychology, 53, 297-303.

MoRI, S. (1998). Effects of stimulus information and number of stimuli on sequential dependencies in absolute identification. Canadian Journal of Experimental Psychology, 52, 72-83.

Mori, S., \& WARD, L. M. (1995). Pure feedback effects in absolute identification. Perception \& Psychophysics, 57, 1065-1079.

PARDuCCI, A. (1965). Category judgment: A range-frequency model. Psychological Review, 2, 407-418.

Petzold, P. (1981). Distance effects on sequential dependencies in categorical judgments. Journal of Experimental Psychology: Human Perception \& Performance, 7, 1371-1385.

Petzold, P. (1982). The edge effect of discriminability in categorical judgments. In H. G. Geissler and P. Petzold (Eds.), Psychophysical judgment and the process of perception (pp. 222-232). Amsterdam: North-Holland.

Petzold, P., \& Haubensak, G. (2000). The influence of category membership of stimuli on sequential effects. Unpublished manuscript.

Petzold, P., \& Haubensak, G. (in press). Local frames of reference: A multiple standards model. In S. Kaernbach (Ed.), Psychophysics beyond sensation. Hillsdale, NJ: Erlbaum.

Schifferstein, H. N. J., \& FriJters, J. E. R. (1992). Contextual and sequential effects on judgments of sweetness intensity. Perception \& Psychophysics, 52, 243-255.

Schifferstein, H. N. J., \& Kuiper, W. E. (1997). Sequence effects in hedonic judgments of taste stimuli. Perception \& Psychophysics, 59, 900-912.

Staddon, J. E. R., King, M., \& Lockhead, G. R. (1980). On sequen- tial effects in absolute judgment. Journal of Experimental Psychology: Human Perception \& Performance, 6, 290-301.

Treisman, M. (1984). A theory of criterion setting: An alternative to the attention band and response ratio hypotheses in magnitude estimation and cross-modality matching. Journal of Experimental Psychology: General, 113, 443-463.

Treisman, M., \& Williams, T. C. (1984). A theory of criterion setting with an application to sequential dependencies. Psychological Review, 91, 68-111.

VolkmanN, J. (1951). Scales of judgment and their implication for social psychology. In J. H. Rohrer \& M. Sherif (Eds.), Social psychology at the crossroads (pp. 279-294). New York: Harper \& Row.

WARD, L. M. (1979). Stimulus information and sequential dependencies in magnitude estimation and cross-modality matching. Journal of Experimental Psychology: Human Perception \& Performance, $\mathbf{5}$, 444-459.

WARD, L. M. (1982). Mixed-modality psychophysical scaling: Sequential dependencies and other properties. Perception \& Psychophysics, 31, 53-62.

WARD, L. M. (1985). Mixed-modality psychophysical scaling: Interand intramodality sequential dependencies as a function of lag. Perception \& Psychophysics, 38, 512-522.

WARD, L. M. (1986). Mixed-modality psychophysical scaling: Double cross-modality matching for "difficult" continua. Perception \& Psychophysics, 39, 407-417.

WARD, L. M., \& LocKHEAD, G. R. (1970). Sequential effects and memory in category judgments. Journal of Experimental Psychology, 84, 27-34.

WARD, L. M., \& LocKheAd, G. R. (1971). Response system processes in absolute judgment. Perception \& Psychophysics, 9, 73-78.

Winer, B. J. (1962). Statistical principles in experimental design. New York: McGraw-Hill.

WitTE, W. (1975). Zum Gestalt- und Systemcharakter psychischer Bezugssysteme. In S. Ertel (Ed.), Gestalttheorie in der modernen Psychologie (pp. 184-202). Darmstadt: Steinkopf.

\section{NOTE}

1. The calculation of mean values and other calculations concerning correlations were performed with $z$-transformed values.

(Manuscript received for publication June 16, 1999; revision accepted for publication January 15, 2001.) 